\title{
Tratamiento farmacológico de la insuficiencia cardíaca. ¿Qué hay de nuevo?
}

\author{
Dr. Michel Komajda
}

\section{Resumen}

El manejo de la insuficiencia cardíaca crónica con fracción de eyección reducida (ICFEr) ha cambiado considerablemente en los últimos 30 años: la introducción de inhibidores de la enzima convertidora de angiotensina (IECA), bloqueadores beta (BB), antagonistas de los receptores de la angiotensina (ARAII), antagonistas de los receptores de los mineralocorticoides (ARM) y del bloqueador de canales If (BCI), ivabradina, han llevado a una reducción significativa en la mortalidad y en la mortalidad por insuficiencia cardíaca (IC).

Recientemente, un ensayo que prueba un doble inhibidor que bloquea el receptor de angiotensina II y la neprilisina, la enzima responsable de la degradación del péptido natriurético tipo B, mostró que esta molécula compleja mejoró los resultados clínicos en comparación con el IECA enalapril.

Sin embargo, persisten retos en en el manejo de esta condición: un cumplimiento subóptimo de las recomendaciones de las guías, un perfil cambiante de pacientes con IC que son mayores y tienen múltiples comorbilidades y una alta tasa de rehospitalizaciones tempranas.

La insuficiencia cardíaca con fracción de eyección preservada (ICFEp), que constituye una proporción significativa de la población con IC, sigue siendo un dilema clínico; ninguna intervención farmacológica ha demostrado hasta el momento resultados beneficiosos convincentes. La heterogeneidad de las poblaciones ensayadas, el papel de las comorbilidades, las dificultades para identificar a los pacientes con ICFEp, así como un desajuste entre los fenotipos clínicos y los tratamientos probados son posibles explicaciones del fracaso en encontrar intervenciones beneficiosas.

En general, el manejo de la IC después del alta sigue siendo fragmentado y se requieren acciones concertadas de todos los profesionales involucrados.

Palabras clave: $\quad$ INSUFICIENCIA CARDÍACA

\section{FARMACOLOGÍA}

\section{Pharmacological management of heart failure: what is new?}

\section{Summary}

The management of chronic heart failure with low ejection fraction has changed considerably over the last thirty years: the introduction of angiotensin converting enzyme inhibitors, beta-blockers, angiotensin receptor blockers, mineralocorticoid receptor antagonists and of the ivabradine If blocker has led to a significant reduction in mortality and in heart failure mortality in this condition.

More recently, a trial testing a dual inhibitor blocking the angiotensin-II receptor and neprylisin, the enzyme responsible for B type natriuretic peptide degradation showed that this complex molecule improved clinical outcomes compared to the ACE-inhibitor enalapril.

However, challenges persist in the management of this condition with a suboptimal implementation of guidelines recommended therapies, a changing profile of heart failure patients who are older and have multiple comorbidities and a high rate of early rehospitalizations for heart failure.

Heart failure with preserved ejection fraction, a significant proportion of the heart failure population, remains a clinical dilemma: no pharmacological intervention has so far demonstrated any convincing benefit on outcomes. Heterogeneity of the populations tested, role of comorbidities, difficulties in identifying patients with $\mathrm{HFpEF}$ as well as a mismatch between the clinical phenotypes and the treatments tested are potential explanations of the failure to find beneficial interventions.

Overall the management of heart failure after discharge remains fragmented and concerted actions of all professionals concerned are needed.

Key words: $\quad$ HEART FAILURE

PHARMACOLOGY

Universidad Pierre y Marie Curie - ICAN - Hospital Pitié-Salpêtrière. Departamento de Cardiología.

Correspondencia: Dr.Michel Komajda. Correo electrónico: michel.komajda@aphp.fr

Traducido del inglés con autorización del autor.

Recibido Set 27, 2017; aceptado Nov 1, 2017 


\section{Introducción}

El manejo farmacológico de la IC ha cambiado considerablemente durante los últimos 30 años con la introducción de las principales clases de fármacos. Se ha demostrado que tanto los IECA, como los BB, los ARAII, los ARM, los BCI y, más recientemente, los inhibidores duales que bloquean los receptores de neprilisina y angiotensina, mejoran la mortalidad y la morbilidad en ensayos clínicos aleatorizados de gran magnitud que incluyen pacientes con IC crónica leve a moderada y fracción de eyección reducida. ${ }^{1-19}$. Sin embargo, hay áreas en las que no existe evidencia o falta el conocimiento a pesar de numerosos intentos de ensayos farmacológicos y no farmacológicos. Esto incluye, en particular, a la IC aguda y a la ICFEp, en las que se ha logrado poco o ningún progreso. Además, las hospitalizaciones prolongadas y recurrentes por IC siguen siendo una carga para los sistemas de atención de la salud debido al costo y a la mala calidad de vida asociados.

El objetivo de este trabajo es revisar los éxitos y fracasos en el manejo de esta patología e identificar los desafíos restantes.

Insuficiencia cardíaca crónica con fracción de eyección reducida. Una historia exitosa La medicina basada en la evidencia en la IC se ha desarrollado esencialmente en pacientes con baja fracción de eyección.

Dos ensayos claves, controlados y aleatorizados, (CONSENSUS y SOLVD), que usaron enalapril en aproximadamente 2.800 pacientes sintomáticos leves, moderados o severos, han demostrado que la adición de un IECA a los diuréticos y, en la mayoría de los casos digoxina y/o espironolactona, redujo significativamente la mortalidad global en un $20 \%{ }^{(1-3)}$. En los pacientes sintomáticos inscritos en el SOLVD hubo también una reducción significativa de las hospitalizaciones por IC.

Finalmente, el beneficio de los IECA en las muertes $u$ hospitalizaciones por IC también se demostró en tres grandes ensayos (SAVE, TRACE, AIRE)(4) que incluían pacientes con IC y / o disfunción ventricular izquierda después de infarto agudo de miocardio $(\mathrm{IAM})^{(4)}$. Estos resultados impresionantes llevaron a la recomendación de utilizar los IECA como terapia de primera línea en la ICFEr en las directrices internacionales ${ }^{(5)}$.

La evidencia que apoya el uso de BB en la misma indicación proviene de los resultados de los grandes ensayos clínicos publicados a finales de los 90 o principios de los 2000: los estudios del grupo US Carvedilol Heart Failure mostraron una reducción en la mortalidad con carvedilol ${ }^{(6)}$. Este programa fue seguido por tres estudios clínicos aleatorizados (CIBIS II (bisoprolol), MERIT HF (succinato de metoprolol CR / XL) y COPERNICUS (carvedilol), que incluyeron a casi 9.000 pacientes y mostraron resultados consistentes con una reducción de la mortalidad de 1/3 y de las hospitalizaciones por IC, en pacientes que ya recibían IECA. Estos resultados positivos fueron confirmados posteriormente en la población de edad avanzada ( $\geq 70$ años) inscrita en el estudio SENIORS, donde el nebivolol redujo significativamente el end point compuesto de muerte $\mathrm{u}$ hospitalización por IC, pero no la mortalidad(7-10).

Estos hallazgos llevaron a la recomendación de usar BB junto con IECA en todos los pacientes con ICFEr.

La evidencia que sustenta el uso de ARM en la IC apareció más temprano en pacientes con IC severa. En el estudio RALES, la espironolactona redujo a 1/3 la mortalidad y la hospitalización por IC en los pacientes severamente sintomáticos (clase III de la NYHA) (11). Sin embargo, en ese momento solo $11 \%$ de los pacientes estaban tratados con BB.

Más recientemente, el ensayo EMPHASIS HF demostró que la adición de eplerenona en pacientes levemente sintomáticos tratados con IECA o ARA II y $\mathrm{BB}$ redujo significativamente en $37 \%$ la mortalidad cardiovascular y las hospitalizaciones por insuficiencia cardíaca y otros end points clínicos $^{(12)}$.

Por lo tanto, las guías de la Sociedad Europea de Cardiología (ESC) recomiendan considerar esta clase de fármaco en todos los pacientes con ICFEr y con síntomas persistentes a pesar de los IECA o ARA II y BB.

Se recomienda el uso de ARA II como alternativa a los IECA en pacientes intolerantes a esta clase después de la publicación de la alternativa CHARM que demostró que, en estos pacientes, el candesartán redujo la mortalidad cardiovascular o la IC en un $23 \%{ }^{(13)}$.

Dos ensayos adicionales, ValHeft (valsartán) y CHARM-added (candesartán), analizaron el beneficio potencial proporcionado por una combinación de un ARA II con un IECA(14,15). Hubo una reducción de la hospitalización por IC y en el CHARM se añadió una reducción de la muerte cardiovascular. Sin embargo, dado que la magnitud del beneficio logrado por esta combinación es menor que el de la combinación de un IECA con un ARM, se indican ahora los ARAII principalmente como una alternativa a los IECA en caso de intolerancia.

La ivabradina es un fármaco que inhibe el canal "funny" (If) en el nódulo sinoauricular y enlentece la frecuencia cardíaca en pacientes en ritmo sinusal. La magnitud de la reducción depende directamente de la frecuencia cardíaca de referencia y es 
más pronunciada en pacientes con una frecuencia cardíaca elevada. Este nuevo fármaco se probó en asociación con IECA / ARAII, BBy, en la mayoría de los casos, un ARM, en un gran ensayo controlado aleatorizado que incluyó pacientes con IC leve a moderada, baja fracción de eyección y ritmo sinusal con frecuencia cardíaca $\geq 70 \mathrm{lpm}^{(16)}$. Hubo una reducción significativa del $18 \%$ en el end point compuesto principal de muerte cardiovascular y hospitalización por IC. La reducción de la muerte cardiovascular o por todas las causas por separado no fue significativa, mientras que las hospitalizaciones por IC se redujeron en un 26\%. También se observó una mejoría significativa en la calidad de vida, así como un efecto de remodelado inverso ${ }^{(17,18)}$.

La ivabradina se recomienda ahora en pacientes con ICFEr sintomáticos en ritmo sinusal $\geq 70 \mathrm{lpm}$ a pesar del tratamiento con IECA/ARAII, ARM y BB a las dosis máximas toleradas (o sin $\mathrm{BB}$ cuando están contraindicados).

Recientemente, un gran ensayo controlado aleatorizado (PARADIGM-HF) evaluó, en una gran población de pacientes con IC leve a moderada, un inhibidor doble (LCZ696-valsartan/sacubitril) que bloquea el receptor de la angiotensina y la neprilisina (enzima que escinde el péptido natriurético tipo B haciéndolo inactivo) comparándolo con $20 \mathrm{mg}$ de enalapril(19). El ensayo se interrumpió prematuramente debido al beneficio: hubo una reducción significativa del $20 \%$ del criterio combinado de mortalidad cardiovascular y hospitalización por IC, de mortalidad cardiovascular sola $(20 \%)$ (que fue el desencadenante predefinido para recomendar la terminación temprana), así como de todas las causas de muerte (16\%) y de hospitalizaciones por IC (21\%). En general, la seguridad fue buena: hubo más episodios de hipotensión, pero la tolerancia renal fue mejor y la tos fue significativamente menos frecuente que con el enalapril; el número de angioedemas, un efecto secundario bien conocido de los bloqueantes de la neprilisina relacionado con el aumento de la concentración plasmática de bradiquinina, fue sólo ligeramente mayor que en el grupo control.

Tanto la Administración estadounidense de Alimentos y Medicamentos (FDA) como la Agencia Europea de Medicamentos (EMA) han convalidado la indicación del sacubitril/valsartan en ICFEr que permanece sintomática bajo terapia médica óptima. El problema es definir con mayor precisión la población que se beneficia de esta nueva clase de medicación. El sacubitril/valsartan no debe utilizarse en pacientes que no hayan sido tratados previamente con IECA o con ARA II ya que prácticamente todos los inscritos en PARADIGM HF fueron tratados previamente con cualquiera de estas dos clases; deberían reservarse entonces a pacientes con IC crónica que permanezcan sintomáticos o que muestren evidencia de deterioro de la función cardíaca bajo terapia óptima con las drogas modificadoras de la enfermedad previamente mencionadas (IECA/ARA II,BB, ARM).

Dado que este fármaco es un vasodilatador potente, no debe prescribirse en pacientes con presión arterial sistólica baja: <100 mmHg. De igual manera, una kalemia $>5,4 \mathrm{mEq} / \mathrm{l}$ es una contraindicación porque la dosis de valsartán contenida en $200 \mathrm{mg}$ de este nuevo compuesto es equivalente a $320 \mathrm{mg}$ como medicación independiente.

Otro aspecto a considerar es el de la fijación de precios y el reembolso de la droga que siguen siendo objeto de debate en varios países.

Las guías de la ESC de 2016 recomiendan sacubitril/valsartan e ivabradina como terapia de tercera línea después de los IECA o ARA II, BB y ARM en pacientes que permanecen o están en ritmo sinusal con una frecuencia cardíaca de $70 \mathrm{lpm}$ o más (ivabradina $)^{(5)}$. Estas pautas también establecen que estos nuevos fármacos pueden usarse simultáneamente si el perfil del paciente corresponde a las indicaciones.

El progreso logrado en la farmacología ha mejorado indudablemente los resultados de la ICFEr. Hace veinte años, la tasa de mortalidad anual era de $16 \%$ en el grupo de placebo de SOLVD. En el estudio SHIFT publicado en 2010, la tasa había descendido al 9\%. La tasa de mortalidad por IC se redujo a la mitad entre los dos ensayos. La diferencia no se observa sólo en las poblaciones inscritas en los ensayos clínicos, que por definición son altamente seleccionadas. Un análisis de la mortalidad directamente relacionada con la IC en ocho países europeos muestra que ha estado disminuyendo constantemente en los últimos quince años ${ }^{(20)}$.

Sin embargo, la mortalidad por todas las causas sigue siendo muy alta después de una hospitalización por IC aguda: en el Euro Observational Study on Heart Failure, la tasa de mortalidad anual fue del $17 \%$ y la causa fue cardiovascular en el $60 \%$ de los $\operatorname{casos}^{(21)}$.

\section{El desafío de las hospitalizaciones y rehospitalizaciones}

El hecho de que la mejor sobrevida y la modificación de la enfermedad por los medicamentos no siempre se traducen en una reducción de las hospitalizaciones y, sobre todo, de rehospitalizaciones tempranas 
en la población general, es un motivo de preocupación.

Aunque los datos recientes de hospitalizaciones por IC en los resúmenes de alta hospitalaria en los Estados Unidos, muestran que han disminuido con el tiempo, sigue siendo la principal causa de derivación al hospital, como lo demuestra un análisis de hospitalizaciones en $\mathrm{Suecia}^{(22,23)}$.

La fase inicial de cuatro a seis semanas después del alta es una "fase vulnerable" que está asociada con una alta tasa de mortalidad y rehospitalizaciones ${ }^{(24)}$.

El riesgo de morir en los primeros treinta días después de una hospitalización se incrementa seis veces en comparación con los pacientes no hospitalizados. Del mismo modo, en el estudio EVEREST, el $25 \%$ de los pacientes se rehospitalizaron durante el mismo período de tiempo después de una IC descompensada ${ }^{(25)}$. En un análisis de los resúmenes de alta hospitalaria en los Estados Unidos, la IC fue la primera causa de hospitalizaciones recurrentes ${ }^{(26)}$.

Un análisis en pacientes ancianos hospitalizados por IC en los Estados Unidos sugiere una tendencia bidireccional con una reducción en la mortalidad posalta, pero un aumento en las tasas de rehospitalización precoz ${ }^{(27)}$. Dado que las hospitalizaciones representan el $70 \%$ de los gastos relacionados con el manejo de esta enfermedad, no es sorprendente que el costo del tratamiento se duplicará entre 2010 y 2030 en los Estados Unidos ${ }^{(28)}$.

Por otra parte, el manejo específico de la IC aguda no ha cambiado en los últimos veinte años. Un número de ensayos que utilizan fármacos muy diferentes, tales como nuevos inotrópicos, antagonistas de la arginina-vasopresina, neseritide, antagonistas del receptor A1 de adenosina o antagonistas del receptor de la endotelina, no han demostrado ninguna mejoría en los resultados a corto o mediano plazo y las Guías de la ESC aún recomiendan los diuréticos intravenosos, el oxígeno, los opioides si son necesarios o los "viejos" inotrópicos, ya que juegan un papel central en el manejo de esta condición. Recientemente, la decepción vino de la mano de dos ensayos (TRUE HF; RELAX AHF 2) que testearon un péptido natriurético como el ularitide y la droga serelaxina, un vasodilatador con propiedades pleiotrópicas, y que no mostraron ningún beneficio en los end points clínicos duros y sólo una mejoría, clínicamente cuestionable en los síntomas intrahospitalarios ${ }^{(29,30)}$.

Pueden proponerse varias explicaciones para entender la explosión de rehospitalizaciones relacionadas con la IC: 1) Los pacientes que murieron previamente ahora sobreviven a expensas de un aumento en la tasa de rehospitalizaciones. 2) El perfil cambiante de los pacientes que son más añosos y tienen frecuentemente múltiples comorbilidades puede hacer que el manejo sea más complejo debido a posibles contraindicaciones o intolerancias, existiendo además, mayor riesgo de rehospitalización debido a una mayor fragilidad. 3) Utilización subóptima de fármacos que salvan vidas, lo que se observa en todos los registros. Encuestas recientes sugieren consistentemente que en el mejor de los casos del 25 al 30\% de los pacientes alcanzan la dosis objetivo de BB. 4) Se observa con frecuencia una cadena asistencial fragmentada cuando los pacientes salen del hospital. Esto se traduce en una brecha en el seguimiento de la atención después del alta y muchos no ven a ningún profesional de la salud durante un largo período de tiempo. En una encuesta nacional realizada en Francia sólo el 29\% de los pacientes había consultado a un cardiólogo en los primeros tres meses después del alta hospitalaria ${ }^{(31)}$.

Las soluciones a esta situación preocupante incluyen mejores esquemas de educación para que todos los pacientes con IC sean conscientes de los objetivos del tratamiento, de los síntomas de descompensación, de los efectos secundarios más comunes, de los medicamentos utilizados y de las necesidades dietéticas. Este enfoque ha demostrado ser eficaz, pero es limitado en los ancianos con trastornos cognitivos en los que el papel de la familia se vuelve crucial.

La medición de biomarcadores en forma seriada es otra manera de abordar esta cuestión y un metaanálisis reciente ha demostrado que la estrategia guiada por péptidos natriuréticos reduce las reinternaciones en un $25 \%{ }^{(32)}$. Sin embargo, se observó la magnitud del beneficio sobre la mortalidad sólo en menores de 75 años, mientras que no hubo beneficio en todas las causas de muerte en pacientes de edad avanzada, que representan la mayoría de la población con IC. La telemedicina basada en la estructura de llamadas telefónicas o control a distancia monitorizando biomarcadores es otra herramienta para mejorar los resultados y reducir las hospitalizaciones, aunque no todos los estudios han mostrado beneficio ${ }^{(33)}$. Una limitación para su desarrollo es que, generalmente, esta tarea no es reembolsable y, por lo tanto, el tiempo utilizado no crea ninguna ganancia, con la limitante real que eso genera respecto a los recursos humanos.

Insuficiencia cardiaca con fracción de eyección preservada: un dilema clínico A diferencia de lo observado en la ICFEr, todos los intentos para reducir la mortalidad y morbilidad en la ICFEp han fracasado. 
Un estudio (PEP CHF) testeó el IECA perindropil en una población añosa de 850 pacientes y falló en demostrar beneficio en el end point primario de cualquier causa de muerte y hospitalizaciones por IC (34). Dos estudios testearon un ARA II: el CHARM-PRESERVED evaluó el candesartán en 3.023 pacientes y el I-PRESERVED el irbesartán en 4.128 pacientes añosos ${ }^{(35,36)}$.

En el CHARM-PRESERVED, hubo una tendencia no significativa para la reducción del riesgo del end point primario de muerte cardiovascular y hospitalizaciones por IC, que estuvo dada por la reducción de las hospitalizaciones. En el I-PRESERVE, no hubo una reducción significativa en el end point compuesto de todas las causas de muerte y hospitalizaciones cardiovasculares y ningún objetivo secundario mostró tendencia a favor de irbesartán. Recientemente, el ensayo TOPCAT, que probó la espironolactona, mostró sólo una reducción del 11\% del riesgo relativo del end point primario compuesto de muerte cardiovascular, hospitalización por IC o paro cardíaco abortado(37). Las hospitalizaciones fueron el único componente del end point primario que mostró una reducción significativa y el ensayo fue criticado debido a las enormes variaciones geográficas en las tasas de eventos.

El estudio SUPPORT evaluó la adición de un ARA II, el olmesartán, a la terapia existente, en una gran población de pacientes con hipertensión, IC estable y fracción de eyección preservada ${ }^{(38)}$. Se concluyó que la adición del ARA II no se asoció con mejores resultados, se registraron más episodios de insuficiencia renal y, además, la combinación de un IECA, olmesartán y un BB se asoció con más eventos cardiovasculares.

Estos resultados son preocupantes en la medida que este subconjunto de pacientes representa una parte creciente de la población total de pacientes con $\mathrm{IC}^{(39)}$.

Varios factores pueden explicar los resultados decepcionantes observados en los grandes ensayos clínicos aleatorizados, así como en los estudios de prueba de concepto:

a) Los pacientes con ICFEp suelen ser más añosos y tienen múltiples comorbilidades que vuelven más complicado su manejo ${ }^{(40,41)}$.

b) Posiblemente en relación con mayor comorbilidad no cardíaca, la proporción relativa de muerte no cardiovascular es mayor en la ICFEp que en la $\mathrm{ICFEr}^{(42)}$.

c) El diagnóstico de la ICFEp es desafiante ya que requiere la combinación de signos y síntomas de
IC, una fracción de eyección "preservada" $\geq 50 \%$ y evidencia objetiva de distensibilidad anormal con alteración en el llenado o relajación del ventrículo izquierdo ${ }^{(5)}$.

El componente de relajación o distensibilidad anormal puede ser difícil de probar; en su valoración se le da un lugar central a la relación e/e' en el eco-Doppler y a otros marcadores alternativos que incluyen el agrandamiento auricular izquierdo, la hipertrofia ventricular izquierda o niveles elevados de péptidos natriuréticos. Existe cierta preocupación de que algunos pacientes inscritos en los ensayos clínicos sobre ICFEp no tuvieran realmente IC, sino que simplemente tuvieran hipertrofia ventricular izquierda $\mathrm{y}$ causas no cardíacas para la disnea, como la enfermedad pulmonar crónica o la obesidad. Sin embargo, la tasa de mortalidad cardiovascular y hospitalizaciones por IC es sin duda mayor en los ensayos de ICFEp que en aquellos de pacientes con hipertensión arterial con o sin hipertrofia ventricular izquierda ${ }^{(43)}$.

d) Otra causa de fracaso es la heterogeneidad de los pacientes incluidos en los grandes ensayos clínicos con respecto a la edad o los niveles plasmáticos de péptidos natriuréticos, lo que sugiere que estos pacientes se encuentran en diferentes etapas de su enfermedad.

e) También desempeñan un papel en estos resultados, factores relacionados con los ensayos, como las dificultades para reclutar pacientes lo que conduce a períodos prolongados de reclutamiento con el consiguiente abandono y disminución de publicaciones.

f) Finalmente, los factores relacionados con la droga pueden jugar también un papel. La comprensión de la fisiopatología de la ICFEp sigue siendo subóptima y un enfoque uniforme de su manejo puede no ser adecuado ya que se han descrito varios fenotipos de esta condición ${ }^{(44,45)}$.

El fracaso del ensayo RELAX para demostrar cualquier beneficio del sildenafilo, el inhibidor de la fosfodiesterasa-5, puede atribuirse al hecho de que estos pacientes no tenían hipertensión pulmonar a diferencia de lo que se requería en pequeños ensayos previos o al hecho de que el defecto fisiopatológico no es un aumento de la degradación de GMPc, sino más bien una producción insuficiente ${ }^{(46)}$. Sin embargo, el ensayo SOCRATES- Preserved que probó el vericiguat, un activador soluble de la guanilato-ciclasa no mostró ningún beneficio en los criterios clínicos; y la ivabradina, probada en un ensayo de prueba de con- 
Tabla 1. Clases de fármacos utilizados y efectos comprobados en la insuficiencia cardíaca con fracción de eyección preservada (ICFEp) y reducida (ICFEr)

\begin{tabular}{|c|c|c|}
\hline Clase & $\begin{array}{c}\text { ICFER } \\
\text { Efectos en morbimortalidad }\end{array}$ & $\begin{array}{c}\text { ICFEP } \\
\text { Efectos en morbimortalidad }\end{array}$ \\
\hline Inhibidores ECA & $\stackrel{+}{\stackrel{+}{(\text { CONSENSUS-SOLVD) }}}$ & $\stackrel{-}{-}$ \\
\hline ARA II & $\stackrel{+}{+}$ & $\begin{array}{c}- \\
(\text { I-PRESERVED) } \\
\pm \text { (ingresos por IC) } \\
\text { (CHARM-preservad) }\end{array}$ \\
\hline Betabloqueantes & $\begin{array}{c}+ \\
\text { (programa US } \\
\text { CARVEDILOL-CIBIS II- } \\
\text { MERIT HF-COPERNICUS }\end{array}$ & $\begin{array}{c}\stackrel{ \pm}{(\text { SENIORS) }} \\
\text { (S) }\end{array}$ \\
\hline ARM & $\stackrel{+}{\stackrel{+}{(R A L E S-~ E M P H A S I S) ~}}$ & $\stackrel{ \pm}{ \pm}$ (ingresos por IC-TOPCAT) \\
\hline Ivabradina & $\begin{array}{c}+ \\
(\text { SHIFT) }\end{array}$ & - \\
\hline LCZ696 & $\stackrel{+}{\stackrel{+}{(\text { PARADIGM HF })}}$ & $?$ \\
\hline Digoxina & $\begin{array}{c}\stackrel{ \pm}{\text { (ingresos por IC -DIG) }} \\
\text { (i) }\end{array}$ & - \\
\hline Diuréticos & $?$ & $?$ \\
\hline
\end{tabular}

\section{Resumen conceptual*}

- El manejo de la insuficiencia cardíaca ha cambiado considerablemente durante los últimos 30 años con la amplia difusión de las terapias modificadoras de la enfermedad.

- El progreso realizado se ha relacionado exclusivamente con la insuficiencia cardíaca crónica con fracción de eyección reducida y se traduce en una disminución general de la mortalidad y de las hospitalizaciones.

- Sin embargo, la tasa de hospitalizaciones y particularmente de rehospitalizaciones permanece estable.

- No se ha avanzado en la insuficiencia cardíaca con fracción de eyección preservada y en insuficiencia cardíaca aguda.

- Debido al costo del manejo de la IC y a su alta prevalencia en los ancianos, es necesario desarrollar nuevos conceptos y terapias, mejorar la implementación de medicamentos que salvan vidas y coordinar mejor la acción de los profesionales involucrados en su manejo incluyendo cardiólogos, médicos generales, enfermeras y dietistas.

* Realizado por Editor invitado cepto, EDIFY, no mejoró ninguno de los tres end points coprimarios incluyendo cambios en NT-proBNP, distancia de 6 minutos a pie y la relación e/e' en la ecografía Doppler( ${ }^{(47,48)}$.

El ensayo PARAMOUNT probó el doble inhibidor LCZ 696 en un grupo de 266 pacientes y mostró que en comparación con valsartán hubo un cambio favorable en NT-proBNP a las 12 semanas $^{(49)}$. En base a estos resultados preliminares y en los resultados positivos que mostró el estudio PARADIGM HF en pacientes con ICFEr, se ha iniciado el estudio PARAGON con esta nueva molécula en una gran población de pacientes con ICFEp.

En resumen, la ICFEp sigue siendo una condición compleja, con fisiopatología mal entendida, diagnóstico difícil y fenotipo heterogéneo, factores todos ellos, que dificultan su manejo y explican por qué no se ha avanzado en la mejoría de los resultados ${ }^{(50)}$.

\section{Conflicto de intereses}

Michel Komajda ha recibido honorarios por asesoramiento, de las siguientes empresas: Servier, Amgen, Bristol Myers Squibb, Novartis y por conferencias de las siguientes empresas: Servier, Bristol Myers Squibb, Merck Sharp \& Dohme, Astra Zeneca, Menarini, Sanofi, Novartis. 


\section{Bibliografía}

1. Consensus Trial Study Group. Effects of enalapril on mortality in severe congestive heart failure: results of the Cooperative North Scandinavian Enalapril Survival Study (CONSENSUS). N Engl J Med 1987; 316(23):1429-35.

2. SOLVD Investigators, Yusuf S, Pitt B, Davis CE, Hood WB, Cohn JN. Effect of enalapril on survival in patients with reduced left ventricular ejection fractions and congestive heart failure. N Engl J Med 1991; 325(5):293-302.

3. Garg R, Yusuf S. Overview of randomized trials of angiotensin-converting enzyme inhibitors on mortality and morbidity in patients with heart failure. JAMA 1995; 273(18):1450-6.

4. Flather MD, Yusuf S, Køber L, Pfeffer M, Hall A, Murray G, et al. Long-term ACE-inhibitor therapy in patients with heart failure or left-ventricular dysfunction: a systematic overview of data from individual patients. Lancet 2000; 355(9215):1575-81.

5. Ponikowski P, Voors AA, Anker SD, Bueno H, Cleland JG, Coats AJ, et al. 2016 ESC Guidelines for the diagnosis and treatment of acute and chronic heart failure: The Task Force for the diagnosis and treatment of acute and chronic heart failure of the European Society of Cardiology (ESC). Developed with the special contribution of the Heart Failure Association (HFA) of the ESC. Eur J Heart Fail 2016; 18(8):891-975.

6. Packer M, Bristow MR, Cohn JN, Colucei WS Fowler MB, Gilbert EM, et al. The effect of carvedilol on morbidity and mortality in patients with chronic heart failure. U.S. Carvedilol Heart Failure Study Group. N Engl J Med 1996; 334(21):1349-55

7. CIBIS-II Investigators and Committees. The Cardiac Insufficiency Bisoprolol Study II (CIBIS-II): a randomised trial. Lancet 1999; 353(9146):9-13.

8. Hjalmarson A, Goldstein S, Fagerberg B, Wedel H, Waagstein F, Kjekshus J, et al. Effects of controlled-release metoprolol on total mortality, hospitalizations, and well-being in patients with heart failure: the Metoprolol CR/XL Randomized Intervention Trial in congestive heart failure (MERIT-HF). JAMA 2000; 283(10):1295-302

9. Krum H, Roecker EB, Mohacsi P, Rouleau JL, Tendera M, Coats AJ, et al. Effects of initiating carvedilol in patients with severe chronic heart failure: results from the COPERNICUS Study. JAMA $2003 ; 289(6): 712-8$

10. Flather MD, Shibata MC, Coats AJ, Van Veldhuisen DJ, Parkhomenko A, Borbola J, et al. Randomized trial to determine the effect of nebivolol on mortality and cardiovascular hospital admission in elderly patients with heart failure (SENIORS). Eur Heart J 2005; 26(3):215-25
11. Pitt B, Zannad F, Remme WJ, Cody R, Castaigne A, Perez A, et al. The effect of spironolactone on morbidity and mortality in patients with severe heart failure. Randomized Aldactone Evaluation Study Investigators. N Engl J Med 1999; 341(10):709-17.

12. Zannad F, McMurray JJ, Krum H, van Veldhuisen DJ, Swedberg K, Shi H, et al. Eplerenone in patients with systolic heart failure and mild symptoms. N Engl J Med 2011; 364(1):11-21.

13. Granger CB, McMurray JJ, Yusuf S, Held P, Michelson EL, Olofsson B, et al. Effects of candesartan in patients with chronic heart failure and reduced left-ventricular systolic function intolerant to angiotensin-converting-enzyme inhibitors: the CHARM-Alternative trial. Lancet 2003; 362(9386): $772-6$.

14. Maggioni AP, Anand I, Gottlieb SO, Latini R, Tognoni G, Cohn JN, et al. Effects of valsartan on morbidity and mortality in patients with heart failure not receiving angiotensin-converting enzyme inhibitors. J Am Coll Cardiol 2002; 40(8):1414-21.

15. McMurray JJ, Ostergren J, Swedberg K, Granger CB, Held P, Michelson EL, et al. Effects of candesartan in patients with chronic heart failure and reduced left-ventricular systolic function taking angiotensin-converting-enzyme inhibitors: the CHARM-Added trial. Lancet 2003; 362(9386): 767-71.

16. Swedberg K, Komajda M, Böhm M, Borer JS, Ford I, Dubost-Brama A, et al. Ivabradine and outcomes in chronic heart failure (SHIFT): a randomised placebo-controlled study. Lancet 2010; 376(9744):875-85.

17. Ekman I, Chassany O, Komajda M, Böhm M, Borer JS, Ford I, et al. Heart rate reduction with ivabradine and health related quality of life in patients with chronic heart failure: results from the SHIFT study. Eur Heart J 2011; 32(19):2395-404.

18. Tardif JC, O'Meara E, Komajda M, Böhm M, Borer JS, Ford I, et al. Effects of selective heart rate reduction with ivabradine on left ventricular remodelling and function: results from the SHIFT echocardiography substudy. Eur Heart J 2011; 32(20):2507-15 .

19. McMurray JJ, Packer M, Desai AS, Gong J, Lefkowitz MP, Rizkala AR, et al. Angiotensin-neprilysin inhibition versus enalapril in heart failure. N Engl J Med 2014; 371(11):993-1004.

20. Laribi S, Aouba A, Nikolaou M, Lassus J, Cohen-Solal A, Plaisance $\mathbf{P}$, et al. Trends in death attributed to heart failure over the past two decades in Europe. Eur J Heart Fail 2012; 14(3):234-9.

21. Maggioni AP, Dahlström U, Filippatos G, Chioncel O, Leiro MC, Drozdz J, et al. EURObservational Research Programme: the Heart 
Failure Pilot Survey (ESC-HF Pilot). Eur J Heart Fail 2010; 12(10):1076-84.

22. Krumholz HM, Normand SL, Wang Y. Trends in hospitalizations and outcomes for acute cardiovascular disease and stroke, 1999-2011. Circulation 2014; 130(12):966-75.

23. Stewart S, Ekman I, Ekman T, Odén A, Rosengren A. Population impact of heart failure and the most common forms of cancer: a study of 1162309 hospital cases in Sweden (1988 to 2004). Circ Cardiovasc Qual Outcomes 2010; 3(6):573-80.

24. Solomon SD, Dobson J, Pocock S, Skali H, McMurray JJ, Granger CB, et al. Influence of nonfatal hospitalization for heart failure on subsequent mortality in patients with chronic heart failure. Circulation 2007; 116(13):1482-7.

25. O'Connor CM, Miller AB, Blair JE, Konstam MA, Wedge P, Bahit MC, et al. Causes of death and rehospitalization in patients hospitalized with worsening heart failure and reduced left ventricular ejection fraction: results from Efficacy of Vasopressin Antagonism in Heart Failure Outcome Study with Tolvaptan (EVEREST) program. Am Heart J 2010; 159(5):841-849.e1.

26. Jencks SF, Williams MV, Coleman EA. Rehospitalizations among patients in the Medicare fee-for-service program. N Engl J Med 2009; 360(14): 1418-28.

27. Heidenreich PA, Sahay A, Kapoor JR, Pham MX, Massie B. Divergent trends in survival and readmission following a hospitalization for heart failure in the Veterans Affairs health care system 2002 to 2006. J Am Coll Cardiol 2010; 56(5):362-8.

28. Heidenreich PA, Trogdon JG, Khavjou OA, Butler J, Dracup K, Ezekowitz MD, et al. Forecasting the future of cardiovascular disease in the United States: a policy statement from the American Heart Association. Circulation 2011; 123(8):933-44.

29. Packer M, Holcomb R, Abraham WT, Anker S, Dickstein K, Filippatos G, et al. Rationale for and design of the TRUE-AHF trial: the effects of ularitide on the short-term clinical course and long-term mortality of patients with acute heart failure. Eur $J$ Heart Fail 2017; 19(5):673-681.

30. Teerlink JR, Voors AA, Ponikowski P, Pang PS, Greenberg BH, Filippatos G, et al. Serelaxin in addition to standard therapy in acute heart failure: rationale and design of the RELAX-AHF-2 study Eur J Heart Fail 2017; 19(6):800-809.

31. Cohen Solal A, Leurs I, Assyag P, Beauvais F, Clerson P, Contre C, et al. Optimization of heart FailUre medical Treatment after hospital discharge according to left ventricUlaR Ejection fraction: the FUTURE survey. Arch Cardiovasc Dis 2012; 105(6-7):355-65.
32. Troughton RW, Frampton CM, Brunner-La Rocca HP, Pfisterer M, Eurlings LW, Erntell H, et al. Effect of B-type natriuretic peptide-guided treatment of chronic heart failure on total mortality and hospitalization: an individual patient meta-analysis. Eur Heart J 2014; 35(23):1559-67.

33. Inglis SC, Clark RA, McAlister FA, Stewart S, Cleland JG. Which components of heart failure programmes are effective? A systematic review and meta-analysis of the outcomes of structured telephone support or telemonitoring as the primary component of chronic heart failure management in 8323 patients: Abridged Cochrane Review. Eur J Heart Fail 2011; 13(9):1028-40

34. Cleland JG, Tendera M, Adamus J, Freemantle $\mathbf{N}$, Polonski L, Taylor J, et al. The perindopril in elderly people with chronic heart failure (PEP-CHF) study. Eur Heart J 2006; 27(19):2338-45.

35. Yusuf S, Pfeffer MA, Swedberg K, Granger CB, Held P, McMurray JJ, et al. Effects of candesartan in patients with chronic heart failure and preserved left-ventricular ejection fraction: the CHARM-Preserved Trial. Lancet 2003; 362(9386): 777-81.

36. Massie BM, Carson PE, McMurray JJ, Komajda M, McKelvie R, Zile MR, et al. Irbesartan in patients with heart failure and preserved ejection fraction. N Engl J Med 2008; 359(23):2456-67.

37. Pitt B, Pfeffer MA, Assmann SF, Boineau R, Anand IS, Claggett B, et al. Spironolactone for heart failure with preserved ejection fraction. N Engl J Med 2014; 370(15):1383-92.

38. Sakata Y, Shiba N, Takahashi J, Miyata S, Nochioka K, Miura M, et al. Clinical impacts of additive use of olmesartan in hypertensive patients with chronic heart failure: the supplemental benefit of an angiotensin receptor blocker in hypertensive patients with stable heart failure using olmesartan (SUPPORT) trial. Eur Heart J 2015; 36(15):915-23.

39. Owan TE, Hodge DO, Herges RM, Jacobsen SJ, Roger VL, Redfield MM. Trends in prevalence and outcome of heart failure with preserved ejection fraction. N Engl J Med 2006; 355(3):251-9.

40. Chan MM, Lam CS. How do patients with heart failure with preserved ejection fraction die? Eur J Heart Fail 2013; 15(6):604-13.

41. Fonarow GC, Stough WG, Abraham WT, Albert NM, Gheorghiade M, Greenberg BH, et al. Characteristics, treatments, and outcomes of patients with preserved systolic function hospitalized for heart failure: a report from the OPTIMIZE-HF Registry. J Am Coll Cardiol 2007; 50(8):768-77.

42. Zile MR, Gaasch WH, Anand IS, Haass M, Little WC, Miller AB, et al. Mode of death in patients with heart failure and a preserved ejection fraction: results from the Irbesartan in heart failure with pre- 
served ejection fraction study (I-Preserve) trial. Circulation 2010; 121(12):1393-405.

43. Campbell RT, Jhund PS, Castagno D, Hawkins NM, Petrie MC, McMurray JJ. What have we learned about patients with heart failure and preserved ejection fraction from DIG-PEF, CHARM-preserved, and I-PRESERVE? J Am Coll Cardiol 2012; 60(23):2349-56.

44. Shah SJ. Matchmaking for the optimization of clinical trials of heart failure with preserved ejection fraction: no laughing matter. J Am Coll Cardiol 2013; 62(15):1339-42.

45. Guazzi M, Vicenzi M, Arena R, Guazzi MD. Pulmonary hypertension in heart failure with preserved ejection fraction: a target of phosphodiesterase- 5 inhibition in a 1-year study. Circulation 2011; 124(2):164-74.

46. Redfield MM, Chen HH, Borlaug BA, Semigran MJ, Lee KL, Lewis G, et al. Effect of phosphodiesterase-5 inhibition on exercise capacity and clinical status in heart failure with preserved ejection fraction: a randomized clinical trial. JAMA 2013; 309(12):1268-77.
47. Pieske B, Maggioni AP, Lam CSP, Pieske-Kraigher E, Filippatos G, Butler J, et al. Vericiguat in patients with worsening chronic heart failure and preserved ejection fraction: results of the SOluble guanylate Cyclase stimulatoR in heart failurE patientS with PRESERVED EF (SOCRATES-PRESERVED) study. Eur Heart J 2017; 38(15):1119-1127.

48. Komajda M, Isnard R, Cohen-Solal A, Metra M, Pieske B, Ponikowski P, et al. Effect of ivabradine in patients with heart failure with preserved ejection fraction: the EDIFY randomized placebo-controlled trial. Eur J Heart Fail 2017 Apr 30. doi: 10.1002/ejhf.876. [Epub ahead of print].

49. Solomon SD, Zile M, Pieske B, Voors A, Shah A, Kraigher-Krainer E, et al. The angiotensin receptor neprilysin inhibitor LCZ696 in heart failure with preserved ejection fraction: a phase 2 double-blind randomised controlled trial. Lancet 2012; 380(9851):1387-95.

50. Komajda M, Lam CS. Heart failure with preserved ejection fraction: a clinical dilemma. Eur Heart J 2014; 35(16):1022-32. 